\title{
Magnetic field sensor with Terfenol-D thin-film coated FBG
}

\author{
Ricardo M. Silva ${ }^{\mathrm{a}, \mathrm{b}}$, G. Chesini ${ }^{\mathrm{a}, \mathrm{c}}$, C. J. Gouveia ${ }^{\mathrm{a}, \mathrm{d}}$, A. B. Lobo Ribeiro ${ }^{\mathrm{e}}$, \\ O. Frazão ${ }^{a}$, C. M. B. Cordeiro ${ }^{c}$ and P. A. S. Jorge ${ }^{a}$ \\ ${ }^{\mathrm{a}}$ INESC Porto, Rua do Campo alegre 687, 4169-007 Porto, Portugal \\ ${ }^{\mathrm{b}}$ Fac. de Ciências da Universidade do Porto, Rua do Campo Alegre 687, 4169-007 Porto, Portugal \\ "Instituto de Física "Gleb Wataghin", Universidade Estadual de Campinas, UNICAMP, Campinas, SP, Brazil \\ ${ }^{\mathrm{d}}$ Centro de Competências de Ciências Exactas e da Engenharia, Universidade da Madeira, 9000-390 Funchal, Portugal \\ ${ }^{\mathrm{e}}$ Faculty of Heath Sciences, University Fernando Pessoa, Rua Carlos da Maia 296, 4200-150 Porto, Portugal
}

\begin{abstract}
In this work a magnetic field sensor based on an FBG coated with a thin film of Terfenol-D is presented. The sensor was tested with two optical interrogation systems: one, a scanning laser system with a $1 \mathrm{pm}$ resolution, and the other a differential white light interferometer (WLI). The results obtained in the magnetic field range of $20 \mathrm{mT}$ to $100 \mathrm{mT}$, show the possibility of increasing the magnetic field measurement resolution, with temperature fluctuations invariance, by a factor of 4.5 when using the WLI system.
\end{abstract}

Keywords: fiber optic sensors, magnetic field, Terfenol-D, differential WLI.

\section{INTRODUCTION}

The first work combining Terfenol-D with optical fiber sensors to measure static magnetic fields was reported in 2000 by Mora et al [1]. This magnetostrictive sensor was composed by two different alloys, with similar thermal expansion coefficient, allowing this way a temperature compensation scheme. The mechanical expansion of both materials due to temperature and magnetic field variations was detected by the two fiber Bragg gratings (FBGs) attached. The spectral difference between the two Bragg wavelengths was proportional to the amplitude of the magnetostriction, and, the wavelength shift produced by the grating bounded to the non-magnetic alloy was proportional to the temperature variation.

An electric current optical sensor based on a passive prototype magnetostriction device for high power applications was developed and demonstrated by Satpathi et al [2]. The optical current sensor concept was achieved by attaching a piece of Terfenol-D to a FBG, thus producing mechanical strain that was proportional to the magnetic field amplitude. Li et al [3], demonstrated a magnetic field sensor based on dual FBGs consisting essentially in a rod of Terfenol-D attached to the optical fiber. One of the gratings was fixed on both ends of the magnetostrictive alloy, while the other was only attached on one point, with the other end free to move. The configuration of dual FBGs was employed for point measurement reference and for easy temperature compensation.

In 2006, Mora et al [4] presented a fiber optic AC current sensor for high voltage lines based on a uniform FBG mounted on a Terfenol-D piece. An innovative way of processing the optical signal from the sensing head allowed a simultaneous measurement of temperature and AC current. The first physical parameter was coded on wavelength shift, while the second one was coded on the amplitude of the signal. The sensor could also operate at long distances with a capacity to be multiplexed. Also a sensor for simultaneous measurement of AC current and temperature was reported by Reilly et al [5]. The device was composed of a magnetically biased Terfenol-D piece fixed to one FBG. The sensor was capable of measuring $\mathrm{AC}$ current at temperatures from $18^{\circ} \mathrm{C}$ to $90^{\circ} \mathrm{C}$.

A hysteresis compensation technique for of a magnetic field sensor, containing a magnetostrictive alloy device, was presented by Davino et al [6]. Due to the nonlinear effects taking place in such materials the magneto-elastic material was accurately modeled in order to compensate for hysteresis.

In 2009, for the first time a fiber optic magnetic field sensor with a thin film of Terfenol-D instead of the bulk magnetostrictive materials was reported by Yang et al [7]. By magnetron sputtering deposition process, Terfenol-D thin films were deposited on etched FBGs.

Quintero et al [8], presented a sensor for DC and AC magnetic field measurements. The grating was coated with a layer of a composite mixed with particles of Terfenol-D. Different compositions were tested and the best magnetostrictive response was obtained using a 30\% volume fraction of Terfenol-D mixed in a matrix of epoxy resin as blender. The 
effect of a compressive pre-stress in the sensor was also investigated. Recently, Smith et al [9], presented a novel sensor for detect static magnetic fields using a inscribed FBG and a micromachined slot sputtered with Terfenol-D. The grating and the slot were created with femtosecond laser inscribing.

In this work we present a magnetostrictive sensor for DC magnetic fields interrogated by two gratings that measure simultaneously magnetic field and temperature. The magnetic field is measured by a FBGs coated with a thin film of Terfenol-D, while a second uncoated FBG is used for temperature compensation. The sensing head is interrogated using either a scanning laser system or a white light interferometric (WLI) system. Results obtained show that the interferometric readout system allows for temperature independent high resolution measurements.

\section{EXPERIMENTAL RESULTS}

In Figure 1 a) it is presented the experimental configuration for the measurement of the DC magnetic field. For illumination and interrogation of the two FBGs a FS2200 Braggmeter (FiberSensing, SA) was used with a resolution of $1 \mathrm{pm}$ and a spectral range of $100 \mathrm{~nm}$. Around 774 turns of copper wire with $0.75 \mathrm{~mm}$ of diameter were wounded in order to form a solenoid allowing a uniform DC magnetic field with a maximum intensity of $\approx 70 \mathrm{mT}$ to be generated. The FBGs had a central wavelength of $1540 \mathrm{~nm}$ for the magnetic field sensor and $1550 \mathrm{~nm}$ for the temperature sensor, respectively. The FBGs were written in a standard single mode fiber (SMF28) by the phase mask technique with an ultraviolet (UV) excimer laser emitting at $248 \mathrm{~nm}$. The Terfenol-D film was deposited onto the fiber using a BAE-Balzers sputtering process. During characterization of the sputtering system, a plane glass surface was used and the deposition rate was found to be $60 \mathrm{~nm} / \mathrm{min}$. For optical fibers, due to their geometry, the deposition rate slightly differs and was determined to be $40 \mathrm{~nm} / \mathrm{min}$. When using optical fibers, a four-step process was employed to assure that the film was uniformly deposited. Each step consisted on depositing the Terfenol-D film onto the fiber and rotating it 90 degrees. Thin films with $0.8 \mu \mathrm{m}$ and $1.6 \mu \mathrm{m}$ thickness were produced. The length of the fiber on which the Terfenol-D was deposited was approximately $20 \mathrm{~mm}$ with the grating at the middle point. In Figure $1 \mathrm{~b}$ ) it is exhibited a SEM image showing the cross section of the SMF28 fiber coated with Terfernol-D. The coating process allowed obtaining a film with very uniform thickness. To achieve maximum sensitivity to weak magnetic fields, no mechanical pre-stress was applied.

a)

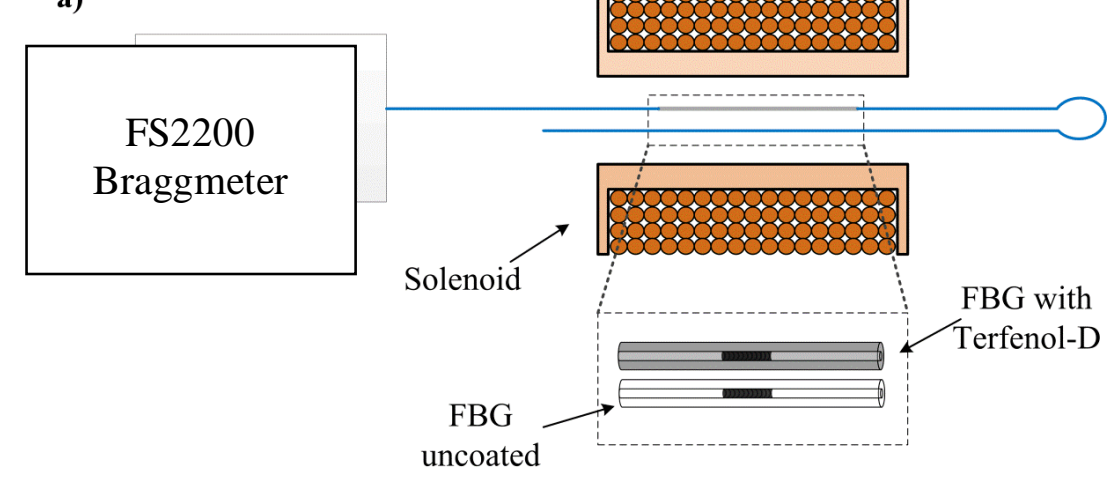

b)

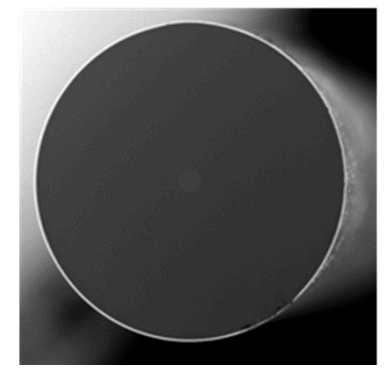

Figure 1 - a) Schematic of the experimental configuration; b) image of the SMF28 coated with the magnetostrictive material. 
Figure 2 shows the response of the grating coated with $1.6 \mu \mathrm{m}$ Terfenol-D thin film to the applied DC magnetic field and the temperature measurement using the uncoated FBG. From the graphic, it is observable that for DC magnetic fields lower than $20 \mathrm{mT}$ the sensing head is insensitive. On the other hand, for DC magnetic fields between $20 \mathrm{mT}$ and $70 \mathrm{mT}$, the sensing head presents a nonlinear response. This result is in agreement with some related works reported earlier, like Quintero et al $[8,10]$. For temperature, the grating was stable during the measurement. In order to detect lower DC magnetic fields, a FBG with increased thickness can be investigated.

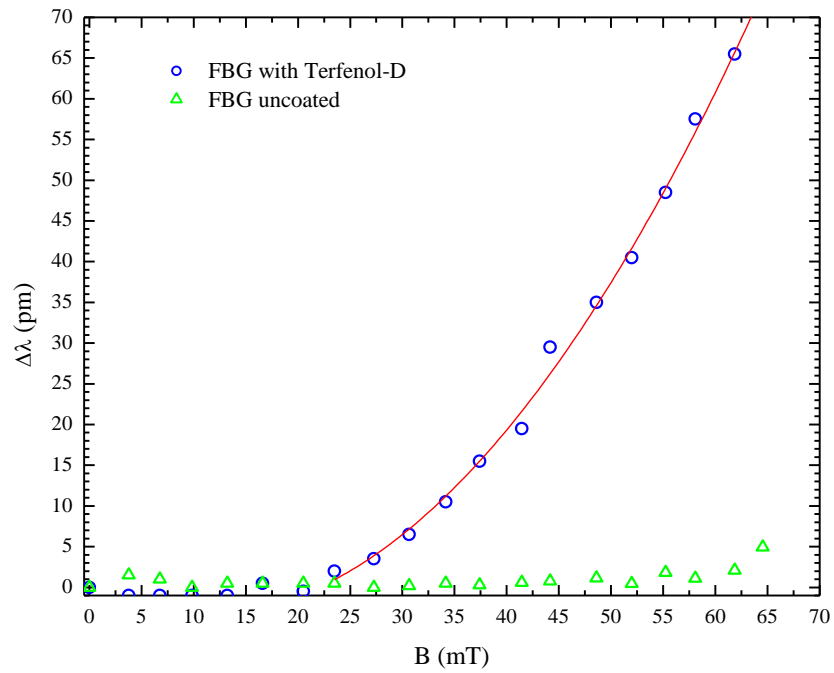

Figure 2 - Response of the FBG coated with $1.6 \mu \mathrm{m}$ Terfenol-D thin film to magnetic field changes and reference temperature measurement as function of the applied magnetic field.

The sensor was also interrogated with a WLI system based in a standard fiber-optic Mach-Zehnder interferometer (MZI). In one of the arms of the MZI, an electro-optical phase modulator (APE from JSDU) was inserted for carrier generation. On the other arm, an air path was implemented using a GRIN lens and a mirror mounted in a translation stage. This allowed to adjust the interferometer path imbalance and also to modulate its phase as desired. A saw tooth modulation $(1 \mathrm{kHz})$ with its amplitude adjusted to obtain a $2 \pi$ phase excursion was used for carrier generation. The system was illuminated by a SLD source $\left(\Delta \lambda=120 \mathrm{~nm}\right.$ and $\left.L_{\text {coherence }}=15 \mu \mathrm{m}\right)$, and used to interrogate the two FBGs connected to its output arms. Using one of the FBGs as reference and the other as sensor, and since the MZI outputs are in phase opposition, most environmentally induce phase drifts can be canceled out, and the system output depends only on the wavelength of the FBGs allowing a very stable and accurate differential phase measurement. Optical circulators routed the FBGs output signals, towards to two photodetectors plugged into a signal acquisition board (DAQ NI 6259 USB). In this situation, after adequate filtering, the output changes in the FBG coated with Terfenol-D arise as a phase shift on the sinusoidal carrier signal, which is retrieved using phase comparison software. To interrogate the FBG coated with Terfenol-D, two different real-time measurements of DC magnetic field were performed using the FS2200 laser scanning system and the WLI, respectively.. In this case, the sensing head was subjected to a uniform magnetic field created by a set of permanent magnets. In order to vary the DC magnetic field we increased the distance between the sensing head and the permanent magnets in stepwise manner. It is important to refer that no mechanical pre-stress was applied. In Figure 3 and Figure 4, the real time response of the DC magnetic field sensor when interrogated with a scanning laser system and the WLI, respectively, can be observed. Comparing these two figures it is evident that using the WLI system the response to DC magnetic field can be tracked with much higher accuracy. The resolution obtained was $1.81 \mathrm{mT}$ and $0.40 \mathrm{mT}$ for the FS2200 system and WLI system, respectively.

\section{CONCLUSIONS}

A sensor based on fiber gratings for detection of temperature and static magnetic fields has been reported. A Terfenol-D thin film was deposited onto the fiber using a sputtering process. The result obtained allows the detection of DC magnetic fields on the order of $20 \mathrm{mT}$. Also, measurements of DC magnetic field were performed using two distinct optical interrogation schemes. A resolution of $1.81 \mathrm{mT}$ was obtained using a $1 \mathrm{pm}$ resolution scanning laser system. The 
use of a WLI system arranged in a differential Mach Zhender configuration allowed to improve system resolution by a factor of 4.5 enabling a resolution of $0.40 \mathrm{mT}$. The use of FBG coated with Terfenol-D thin films with different thickness in combination with interferometric interrogation systems is therefore a promising solution for monitoring of magnetic fields in high power system with no need to etch the optical fiber.

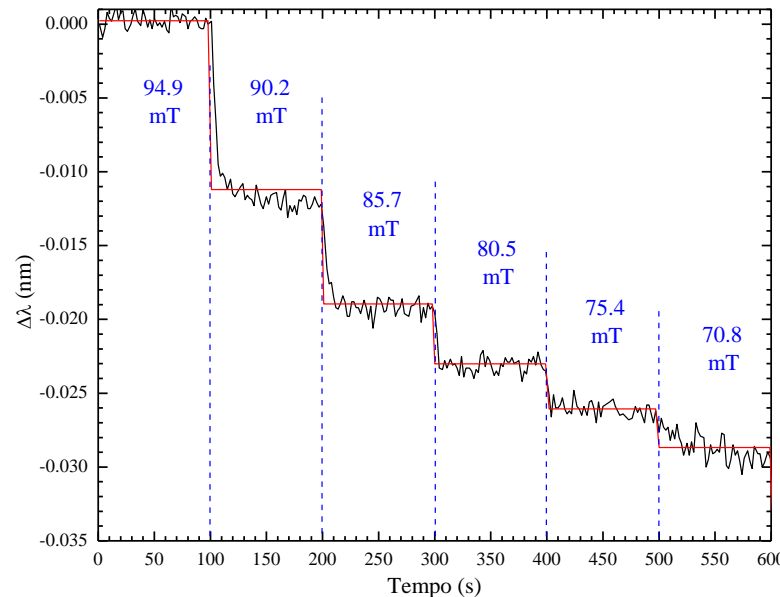

Figure 3 - Sensor response to magnetic field changes when interrogated with a scanning laser system (FS2200).

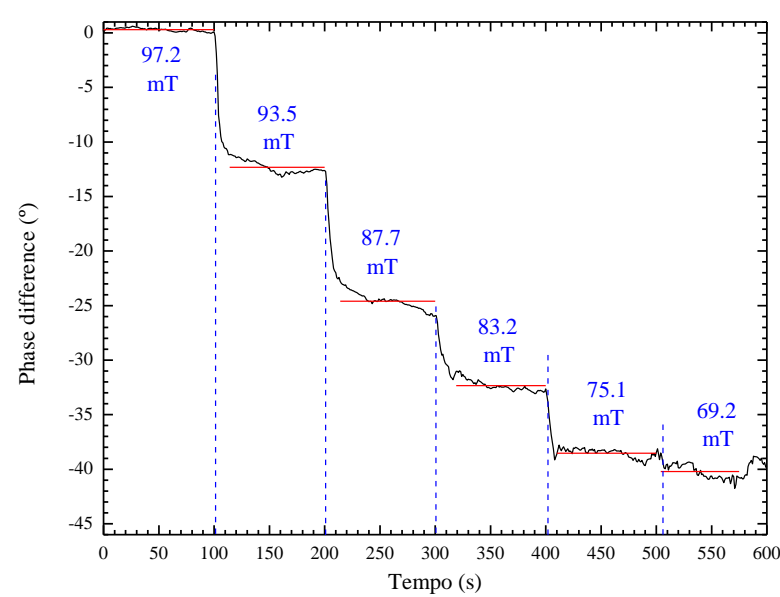

Figure 4 - Sensor response to magnetic field changes when interrogated with a differential WLI system.

\section{ACKNOWLEGMENTS}

This work was supported by ANEEL through the research and development program of the companies of the group TBE -Transmissoras Brasileiras de Energia (EATE -Empresa Amazonense de Transmissão de Energia, ECTE - Empresa Catarinense de Transmissão de Energia, ENTE - Empresa Norte de Transmissão de Energia, ERTE - Empresa Regional de Transmissão de Energia, ETEP - Empresa Paraense de Transmissão de Energia, LUMITRANS- Companhia Transmissora de Energia Electrica and STC - Sistemas de Transmissão Catarinense). The authors also acknowledge the support of cooperation project, CAPES-FCT Brazil/Portugal (\# 293/11, coordinated by authors C.M.B.C. and P. A.S.J.) This work was supported by National Funds through the FCT Fundac ao para a Ci^encia e a Tecnologia (Portuguese Foundation for Science and Technology) within project FCOMP-01-0124-FEDER-022701:

\section{REFERENCES}

[1] J. Mora, A. Diez, J. L. Cruz et al., "A magnetostrictive sensor interrogated by fiber gratings for DC-Current and temperature discrimination," Ieee Photonic Tech L, 12(12), 1680-1682 (2000).

[2] D. Satpathi, J. A. Moore, and M. G. Ennis, "Design of a Terfenol-D based fiber-optic current transducer," Ieee Sens J, 5(5), 1057-1065 (2005).

[3] M. F. Li, et al., "Giant magnetostrictive magnetic fields sensor based on dual fiber Bragg gratings." 490-495.

[4] J. Mora, L. Martinez-Leon, A. Diez et al., "Simultaneous temperature and ac-current measurements for high voltagelines using fiber Bragg gratings," Sensor Actuat a-Phys, 125(2), 313-316 (2006).

[5] D. Reilly, A. J. Willshire, G. Fusiek et al., "A fiber-Bragg-grating-based sensor for simultaneous AC current and temperature measurement," Ieee Sens J, 6(6), 1539-1542 (2006).

[6] D. Davino, C. Visone, C. Ambrosino et al., "Compensation of hysteresis in magnetic field sensors employing Fiber Bragg Grating and magnetoelastic materials," Sensor Actuat a-Phys, 147(1), 127-136 (2008).

[7] M. H. Yang, J. X. Dai, C. M. Zhou et al., "Optical fiber magnetic field sensors with TbDyFe magnetostrictive thin films as sensing materials," Opt Express, 17(23), 20777-20782 (2009).

[8] S. M. M. Quintero, A. M. B. Braga, H. I. Weber et al., “A Magnetostrictive Composite-Fiber Bragg Grating Sensor,” Sensors-Basel, 10(9), 81198128 (2010).

[9] G. N. Smith, et al., "Characterisation and performance of a Terfenol-D coated femtosecond laser inscribed optical fibre Bragg sensor with a laser ablated microslot for the detection of static magnetic fields," Opt Express, 19(1), 363-370 (2011).

[10] S. M. M. Quintero, C. Martelli, A. Braga et al., "Magnetic Field Measurements Based on Terfenol Coated Photonic Crystal Fibers," SensorsBasel, 11(12), 11103-11111 (2011). 